\title{
Caffeic Acid Phenethyl Ester Loaded PLGA Nanoparticles: Effect of Various Process Parameters on Reaction Yield, Encapsulation Efficiency, and Particle Size
}

\author{
Serap Derman \\ Bioengineering Department, Chemical and Metallurgical Engineering Faculty, Yildiz Technical University, \\ Esenler, 34220 Istanbul, Turkey \\ Correspondence should be addressed to Serap Derman; serapacar5@gmail.com
}

Received 22 July 2015; Accepted 13 September 2015

Academic Editor: Ilaria Armentano

Copyright (C) 2015 Serap Derman. This is an open access article distributed under the Creative Commons Attribution License, which permits unrestricted use, distribution, and reproduction in any medium, provided the original work is properly cited.

CAPE loaded PLGA nanoparticles were prepared using the oil in water $(\mathrm{o} / \mathrm{w})$ single emulsion solvent evaporation methods. Five different processing parameters including initial CAPE amount, initial PLGA amount, PVA concentration in aqueous phase, PVA volume, and solvent type were screened systematically to improve encapsulation of hydrophobic CAPE molecule, simultaneously minimize particle size, and raise the reaction yield. Obtained results showed that the encapsulation efficiency of the nanoparticles significantly increased with the increase of the initial CAPE amount $(p<0.05)$ and particle size $(p<0.05)$. Furthermore, the particle size is significantly influenced by initial polymer amount $(p<0.05)$ and surfactant concentration $(p<0.05)$. By the optimization of process parameters, the nanoparticles produced $70 \pm 6 \%$ reaction yield, $89 \pm 3 \%$ encapsulation efficiency, $-34.4 \pm 2.5 \mathrm{mV}$ zeta potential, and $163 \pm 2 \mathrm{~nm}$ particle size with low polydispersity index $0.119 \pm 0.002$. The particle size and surface morphology of optimized nanoparticles were studied and analyses showed that the nanoparticles have uniform size distribution, smooth surface, and spherical shape. Lyophilized nanoparticles with different CAPE and PLGA concentration in formulation were examined for in vitro release at physiological $\mathrm{pH}$. Interestingly, the optimized nanoparticles showed a high (83.08\%) and sustained CAPE release (lasting for 16 days) compared to nonoptimized nanoparticle.

\section{Introduction}

Caffeic acid phenethyl ester (CAPE), a flavonoid-like compound (Figure 1), is one of the most active components of honeybee propolis [1]. Numerous biological and pharmacological effects of CAPE have been reported, such as antiviral [2], antioxidant [3], antiallergic [4], anticarcinogenic [5], anti-inflammatory [6], antimicrobial [7], immunomodulatory $[8]$, and anticancer $[9,10]$ activities. CAPE has no potentially harmful effects on normal cells [11] but also has been shown to inhibit the growth of different types of transformed cells [12]. Due to the inhibiting potential of the transcription factor nuclear factor-kappa B (NF- $\kappa \mathrm{B})$, CAPE has strong antitumor activity in cancer cells $[9,10]$. Miscellaneous study has showed the NF- $\kappa$ B inhibiting potential in different cancer cell lines, including breast cancer MCF-7 [13], malignant peripheral nerve sheath tumors, astrocytoma GRT-MG [14], human B-lymphoma [15], prostate cancer PC-3 [16], myeloid leukemia U-937 [17], and colon cancer HT-29, 26-L5 [18] cells.

On the other hand, CAPE can be hydrolyzed in plasma enzymes by an esterase [19] and this hydrolysis leads to rapid clearance and short half-life and results in poor bioavailability and poor biological performances [20, 21]. Wang et al. reported that the half-life of $5 \mu \mathrm{g} / \mathrm{mL}$ CAPE at $37^{\circ} \mathrm{C}$ was 0.35 hours [22]. Additionally, poorly water soluble character of CAPE limits its in vivo and in vitro efficacy [1].

In the recent years, many strategies have been developed to improve bioavailability and water solubility of hydrophobic drugs including drug carrier systems such as antibodies, liposomes, or nanoparticles [23]. Several disease related drugs/bioactive molecules are successfully encapsulated to improve solubility, bioavailability, and bioactivity $[23,24]$. Among the biocompatible and biodegradable polymeric 


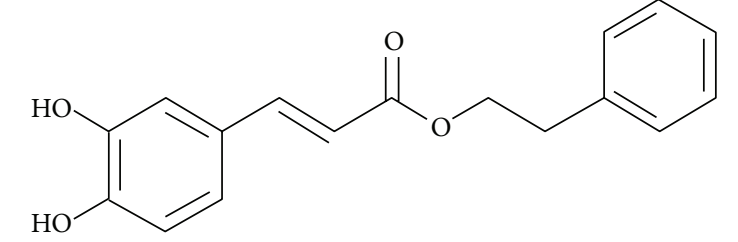

FIGURE 1: Chemical structure of caffeic acid phenethyl ester (CAPE).

nanoparticles PLGA (poly-d,l-lactide-co-glycolide) is one of the most commonly and successfully used biodegradable nanosystems for encapsulation of various therapeutic agents because of its high biodegradability, biocompatibility, and low toxicity [23], and also final degradation products (lactic acid and glycolic acid) are completely safe because they are finally eliminated as carbon dioxide and water [25].

PLGA based polymeric nanoparticles are studied to enhance the biological activity, water solubility, and bioavailability of various drug and natural products because of their small particle size and large surface area [10]. For instance, Singh et al. showed that tea polyphenol loaded PLGA nanoparticles provide 30 -fold higher prevention on the DNA damage than free tee polyphenols [26]. Chaowanachan et al. synthesized efavirenz loaded PLGA nanoparticles and researcher showed that the HIV inhibitory effect of nanoparticle showed up to a 50 -fold reduction in the $50 \%$ inhibitory concentration $\left(\mathrm{IC}_{50}\right)$ compared to free drug [27].

Despite the above positive features of nanoparticle system, there is only one study in the literature concerning encapsulation of CAPE into polymeric nanoparticle. Hyo-Young and colleagues encapsulated CAPE into poly( $\varepsilon$ caprolactone)/poly(ethylene glycol) block copolymer and they showed that CAPE incorporated nanoparticles have superior antimetastatic efficiency against pulmonary metastasis model. However, to the best of our knowledge, there are no studies in the literature about encapsulation of CAPE to PLGA based nanoparticular system.

Thus the first purpose of the present study was to examine the initial CAPE amount, initial PLGA amount, stabilizer concentration in aqueous phase, oil/aqueous phase ratio, and solvent type for optimizing the nanoparticle formulation in respect to reaction yield, encapsulation efficiency, particle size, polydispersity index (PDI), and zeta potential. To synthesize the PLGA nanoparticles in optimum formulation for the first time by oil in water $(\mathrm{o} / \mathrm{w})$ single emulsion solvent evaporation method and detailed characterize improved drug encapsulated nanoparticles were the other aims of the existing study.

\section{Materials and Methods}

2.1. Materials. PLGA (lactide: glycolide $=50: 50$; inherent viscosity $0.45-0.60 \mathrm{dL} / \mathrm{g}, \mathrm{Mw} \sim 38-54 \mathrm{kDa} \mathrm{P} 50 / 50$ ), polyvinyl alcohol, CAPE, acetone, and ethanol were purchased from Sigma Aldrich (St. Louis, USA); dichloromethane (DCM) was obtained from Ridel de Haen. All the chemicals and solvents were of analytical grade and used without further purification. Ultra-pure water was obtained from Millipore MilliQ Gradient system.

The in vitro release measurements were carried out at $37^{\circ} \mathrm{C}$ in phosphate buffer solution at $\mathrm{pH}=7.4$ and $\mathrm{pH}$ 5.2.

2.2. Nanoparticle Preparation Method. The CAPE loaded PLGA nanoparticles were prepared by modified oil in water $(\mathrm{o} / \mathrm{w})$ single emulsion solvent evaporation according to method described by Song et al. [28] with minor modifications. The organic phases consisted of PLGA and CAPE which were dissolved into DCM and ethanol, respectively. Briefly, known amount of CAPE and PLGA (Table 1) was mixed and stirred to ensure that all materials were dissolved. This organic solution was emulsified with the $4 \mathrm{~mL}$ aqueous solution of PVA $(3 \% \mathrm{w} / \mathrm{v})$ by sonication (output power $70 \mathrm{~W}$, power of $80 \%$, and 2 minutes) using a microtip probe sonicator (Bandelin Sonopuls, Germany) over an ice bath. The $\mathrm{o} / \mathrm{w}$ single emulsions were stirred overnight on a magnetic stirrer at room temperature for evaporation of organic phase. The resulting particles were collected by centrifugation at 9.000x rpm for $40 \mathrm{~min}$ (Hettich-Universal $32 \mathrm{R}$ ), washed three times with ultra-pure water to remove excess PVA, and then lyophilized. The free nanoparticles were prepared with similar method without using CAPE and all lyophilized nanoparticles were stored at $-80^{\circ} \mathrm{C}$ until used.

In this study, the effect of various process parameters on reaction yield (RY), encapsulation efficiency (EE), the mean particle size ( $Z$-Ave), polydispersity index (PDI), and zeta potential were investigated, including the initial CAPE amount, initial PLGA amount, PVA concentration in aqueous phase, PVA volume (aqueous-to-organic phase volume ratio) and solvent type (acetone/DCM + ethanol) volume ratio.

\subsection{Characterization of Polymeric Nanoparticles}

2.3.1. Reaction Yield (RY). The nanoparticle preparation technique with a high reaction yield would reduce chemicals loss and product cost. The reaction yield was calculated gravimetrically using the formula given below [29]:

$$
\mathrm{RY}(\%)=\frac{\text { Amount of nanoparticle produced }}{\text { Amount of initial polymer }+ \text { CAPE }} \times 100 \text {. }
$$

2.3.2. Encapsulation Efficiency (EE). The encapsulated CAPE in the NPs was detected in triplicated indirect quantification methods by using UV-Vis Spectroscopy at $323 \mathrm{~nm}$. EE was determined by analyzing the supernatant obtained from the ultracentrifugation of each nanoparticle formulation. CAPE concentration in the supernatant was determined by comparing the concentration to a previously constructed standard calibration curve. The concentration of loading CAPE in nanoparticles was calculated from the total amount of CAPE and the amount of CAPE that was not encapsulated.

The CAPE encapsulation efficiency (EE) was calculated using the formulas given below:

$$
\begin{aligned}
\mathrm{EE}(\%)= & \frac{\text { Amount of CAPE encapsulated in NPs }}{\text { Initial CAPE added }} \\
& \times 100 .
\end{aligned}
$$


TABLE 1: Various process parameters of CAPE loaded NPs.

\begin{tabular}{|c|c|c|c|c|c|c|c|}
\hline \multirow{2}{*}{$\begin{array}{l}\text { Nanoparticle } \\
\text { number }\end{array}$} & \multicolumn{2}{|c|}{ CAPE and PLGA amount } & \multicolumn{2}{|c|}{ PVA } & \multicolumn{3}{|c|}{ Solvent type } \\
\hline & CAPE (mg) & PLGA (mg) & $(\%)$ & $\mathrm{mL}$ & $\begin{array}{c}\text { Aseton } \\
(\mathrm{mL})\end{array}$ & $\begin{array}{c}\text { DCM } \\
(\mathrm{mL})\end{array}$ & $\begin{array}{c}\text { Et-OH } \\
(\mathrm{mL})\end{array}$ \\
\hline NP 1 & 10 & 100 & 3 & 4 & - & 1.5 & 0.5 \\
\hline NP 2 & 20 & 100 & 3 & 4 & - & 1.5 & 0.5 \\
\hline NP 3 & 30 & 100 & 3 & 4 & - & 1.5 & 0.5 \\
\hline NP 4 & 40 & 100 & 3 & 4 & - & 1.5 & 0.5 \\
\hline NP 5 & 50 & 100 & 3 & 4 & - & 1.5 & 0.5 \\
\hline NP 6 & 20 & 100 & 0.1 & 4 & - & 1.5 & 0.5 \\
\hline NP 7 & 20 & 100 & 0.5 & 4 & - & 1.5 & 0.5 \\
\hline NP 8 & 20 & 100 & 1 & 4 & - & 1.5 & 0.5 \\
\hline NP 9 & 20 & 100 & 2 & 4 & - & 1.5 & 0.5 \\
\hline NP 10 & 20 & 100 & 3 & 6 & - & 1.5 & 0.5 \\
\hline NP 11 & 20 & 100 & 3 & 8 & - & 1.5 & 0.5 \\
\hline NP 12 & 20 & 100 & 3 & 10 & - & 1.5 & 0.5 \\
\hline NP 13 & 20 & 100 & 3 & 20 & - & 1.5 & 0.5 \\
\hline NP 14 & 20 & 100 & 3 & 4 & 0.5 & 1 & 0.5 \\
\hline NP 15 & 20 & 100 & 3 & 4 & 0.75 & 0.75 & 0.5 \\
\hline NP 16 & 20 & 100 & 3 & 4 & 1 & 0.5 & 0.5 \\
\hline NP 17 & 20 & 100 & 3 & 4 & 1.5 & - & 0.5 \\
\hline NP 18 & 20 & 200 & 3 & 4 & - & 1.5 & 0.5 \\
\hline NP 19 & 20 & 300 & 3 & 4 & - & 1.5 & 0.5 \\
\hline NP 20 & 20 & 400 & 3 & 4 & - & 1.5 & 0.5 \\
\hline Optimized NP & 50 & 100 & 2 & 4 & - & 1.5 & 0.5 \\
\hline
\end{tabular}

A standard calibration curve of the absorbance as a function of CAPE concentration was studied at $323 \mathrm{~nm}$. All experiments were performed in triplicate.

2.3.3. Mean Particle Size (Z-Ave), Zeta Potential, and Polydispersity Index (PDI). Dynamic light scattering technique was used for determining of the $Z$-average ( $Z$-Ave) and PDI values of nanoparticles using a Zetasizer (Zetasizer Nano ZS, Malvern, UK) instrument equipped with $4.0 \mathrm{mV} \mathrm{He-Ne} \mathrm{laser}$ $(633 \mathrm{~nm})$ [30]. Measurements were carried out in triplicate, at $25 \pm 0.1^{\circ} \mathrm{C}$ with using $0.8872 \mathrm{cP}$ of viscosity and 1.330 of refractive index for the solutions. The number of runs and run durations were chose automatically.

Zeta potential $(\zeta)$ value of nanoparticles was determined by electrophoretic light scattering (ELS) technique and carried out in the folded capillary cell at $25 \pm 0.1^{\circ} \mathrm{C}$ [30]. The measurements were performed in triplicate, with the following parameters: viscosity, $0.8872 \mathrm{cP}$; dielectric constant, 79; $f(k a), 1.50$ (Smoluchowski). The measurement durations and voltage selections were set to automatic mode.

All samples were prepared by diluting with phosphate buffer saline (PBS), filtered with a $0.20 \mu \mathrm{m}$ RC-membrane filter (Sartorius) before measurement.

2.3.4. Scanning Electron Microscopy (SEM). The surface morphology and shape of the CAPE loaded nanoparticles were observed using scanning electron microscopy (SEM) as previously described by Halayqa and Domańska [31]. A drop of nanoparticles suspensions was placed on a black carbon tape with a double side. After drying the samples were coated with gold layer under vacuum and analyzed with SEM (Zeiss EVO LS 10, Germany) at $5 \mathrm{kV}$.

2.3.5. Fourier Transforms Infrared (FT-IR) Spectrometry. IRPrestige 21 FTIR spectrophotometer (Shimadzu, Japan) was used for chemical analyses of the functional groups present in nanoparticles [32]. Measurements were carried out for PLGA, CAPE, and NPs in universal attenuation total reflectance (ATR) mode. The FT-IR spectra were obtained with 16 scans per sample ranging from 4000 to $750 \mathrm{~cm}^{-1}$ and a resolution of $4 \mathrm{~cm}^{-1}$.

2.3.6. In Vitro CAPE Release. The in vitro CAPE release from nanoparticles was studied using a modified dissolution method [33] in phosphate buffer solutions at $\mathrm{pH}$ 7.4. The $\mathrm{pH}$ value was selected to simulate the physiological $\mathrm{pH}$ (7.4) [34]. In a typical release experiment, $5 \mathrm{mg}$ of the CAPE loaded PLGA nanoparticles was suspended in $10 \mathrm{~mL}$ of PBS with $0.01 \%$ sodium azide and the suspension was incubated at $37^{\circ} \mathrm{C}$ in a shaking incubator $(60 \mathrm{rpm})$ at $\mathrm{pH} 7.4$. At selected time intervals (1 hour, 2 hours, 3 hours and 1, 2, 3, 4, 7, 11, 13, and 16 days), the release medium was centrifuged at $9000 \mathrm{rpm}$, $20 \mathrm{~min}$, the supernatant was collected, and the pellet was resuspended with $10 \mathrm{~mL}$ fresh PBS. The CAPE concentration 
TABLE 2: Effect of process parameters on the reaction yield, encapsulation efficiency, mean particle size, PDI, and zeta potential.

\begin{tabular}{|c|c|c|c|c|c|}
\hline Particle number & Reaction yield (\%) & $\begin{array}{l}\text { Encapsulation } \\
\text { efficiency (\%) }\end{array}$ & Size $(\mathrm{nm})$ & PDI & Zeta potential $(\mathrm{mV})$ \\
\hline NP 1 & $39 \pm 7$ & $60 \pm 3$ & $218 \pm 9$ & $0.077 \pm 0.005$ & $-22.4 \pm 0.7$ \\
\hline NP 2 & $40 \pm 6$ & $76 \pm 4$ & $208 \pm 8$ & $0.065 \pm 0.006$ & $-18.7 \pm 0.3$ \\
\hline NP 3 & $48 \pm 7$ & $86 \pm 5$ & $214 \pm 8$ & $0.101 \pm 0.008$ & $-18.1 \pm 1.0$ \\
\hline NP 4 & $49 \pm 6$ & $89 \pm 2$ & $207 \pm 10$ & $0.055 \pm 0.005$ & $-19.2 \pm 1.3$ \\
\hline NP 5 & $53 \pm 5$ & $92 \pm 5$ & $214 \pm 9$ & $0.079 \pm 0.003$ & $-17.7 \pm 1.6$ \\
\hline NP 6 & $74 \pm 7$ & $91 \pm 5$ & $469 \pm 15$ & $0.461 \pm 0.038$ & $-16.3 \pm 1.8$ \\
\hline NP 7 & $80 \pm 8$ & $91 \pm 3$ & $324 \pm 10$ & $0.113 \pm 0.007$ & $-20.2 \pm 3.1$ \\
\hline NP 8 & $64 \pm 6$ & $86 \pm 3$ & $246 \pm 12$ & $0.119 \pm 0.005$ & $-19.6 \pm 0.7$ \\
\hline NP 9 & $63 \pm 9$ & $83 \pm 5$ & $243 \pm 13$ & $0.138 \pm 0.010$ & $-15.8 \pm 1.9$ \\
\hline NP 10 & $68 \pm 8$ & $84 \pm 7$ & $260 \pm 7$ & $0.139 \pm 0.006$ & $-14.0 \pm 2.9$ \\
\hline NP 11 & $70 \pm 7$ & $85 \pm 6$ & $284 \pm 9$ & $0.177 \pm 0.005$ & $-15.6 \pm 1.8$ \\
\hline NP 12 & $68 \pm 6$ & $84 \pm 3$ & $271 \pm 7$ & $0.150 \pm 0.009$ & $-14.2 \pm 1.8$ \\
\hline NP 13 & $64 \pm 8$ & $80 \pm 5$ & $248 \pm 8$ & $0.164 \pm 0.008$ & $-12.7 \pm 1.3$ \\
\hline NP 14 & $15 \pm 4$ & $65 \pm 4$ & $180 \pm 7$ & $0.087 \pm 0.005$ & $-18.7 \pm 2.0$ \\
\hline NP 15 & $7 \pm 4$ & $66 \pm 2$ & $158 \pm 9$ & $0.057 \pm 0.002$ & $-16.6 \pm 1.8$ \\
\hline NP 16 & $12 \pm 6$ & $64 \pm 4$ & $158 \pm 10$ & $0.103 \pm 0.008$ & $-16.2 \pm 2.0$ \\
\hline NP 17 & $15 \pm 7$ & $83 \pm 4$ & $187 \pm 6$ & $0.143 \pm 0.014$ & $-16.3 \pm 2.6$ \\
\hline NP 18 & $83 \pm 6$ & $84 \pm 6$ & $215 \pm 5$ & $0.128 \pm 0.018$ & $-17.5 \pm 1.6$ \\
\hline NP 19 & $86 \pm 7$ & $81 \pm 4$ & $404 \pm 9$ & $0.403 \pm 0.042$ & $-21.4 \pm 2.5$ \\
\hline NP 20 & $92 \pm 5$ & $85 \pm 5$ & $437 \pm 9$ & $0.499 \pm 0.066$ & $-19.4 \pm 2.0$ \\
\hline Optimized NP & $70 \pm 6$ & $89 \pm 3$ & $163 \pm 2$ & $0.119 \pm 0.002$ & $-34.4 \pm 2.5$ \\
\hline
\end{tabular}

in the supernatant was determined with UV-Vis Spectroscopy at $323 \mathrm{~nm}$ by comparing the concentration to a previously constructed standard calibration curve.

2.4. Statistical Analysis. All experiments were repeated at least three times. Data were expressed as mean \pm standard deviation. SPSS 15.0 software was used for statistical analyses [35]. Nonparametric analysis with Mann-Whitney $U$ test was carried out for comparison of the results. $p$ values less than $0.05(p<0.05)$ were considered significant.

\section{Results}

In this study, the o/w single emulsion solvent evaporation method was used for fabrication of CAPE loaded PLGA nanoparticles. The effect of five process parameters on reaction yield (RY), encapsulation efficiency (EE), mean particle size ( $Z$-Ave), polydispersity index (PDI), and zeta potential were investigated. The basic characteristics of the CAPE loaded PLGA nanoparticles were presented in Table 2.

3.1. Effect of Process Parameters on Reaction Yield. High efficiency nanoparticle preparation methods considerably prevent material loss, improve particle production, and decrease manufacturing cost [29]. In this study, all the formulated batches gave wide yields that ranged between $7 \pm$ $4 \%$ and $92 \pm 5 \%$. Obtained results indicated that the reaction yield responses strongly depend on process parameters.
Particularly, initial PLGA and CAPE amount had strongly and significantly positive effects on reaction yield while acetone/DCM + ethanol concentration reduced the reaction yield.

Figure 2(a) shows that the yield rose from $39 \pm 7 \%$ to $53 \pm$ $5 \%$ with the increase in the initial CAPE amount from $10 \mathrm{mg}$ to $50 \mathrm{mg}$.

Additionally, it is clearly seen in Figure 2(b) that initial PLGA amount had a greater effect on the reaction yield when the other system parameters set at a constant value. As shown in Table 2 the reaction yield increased from $40 \pm 6 \%$ to $92 \pm 5$ with the increase in initial PLGA amount from $100 \mathrm{mg}$ to $400 \mathrm{mg}$, indicating that the higher the PLGA amount used in the formulation, the greater the yield. Our results are in agreement with the recent observations of Hussein et al. [29] who found that the reaction yield increased twofold (from $40 \%$ to $80 \%$ ) with a rise in PLGA concentrations from $2.5 \mathrm{mg} / \mathrm{mL}$ to $10 \mathrm{mg} / \mathrm{mL}$. The increase in the PLGA amount gives rise to the viscosity of solutions, causing the formation of larger and denser particles, which will afterward increase the reaction yield $[29,36,37]$.

The effect of PVA volume on the reaction yield was shown in Figure 2(c). The reaction yield first increased up to $68 \pm$ 8 (for $6 \mathrm{~mL}$ of PVA), then reached a plateau, and slowly decreased with an increase in PVA volume.

Figure 2(d) shows the effect of PVA concentration in the aqueous phase on the reaction yield. As depicted from the figure, at low PVA concentration $(<1 \mathrm{w} / \mathrm{v})$ the increase 


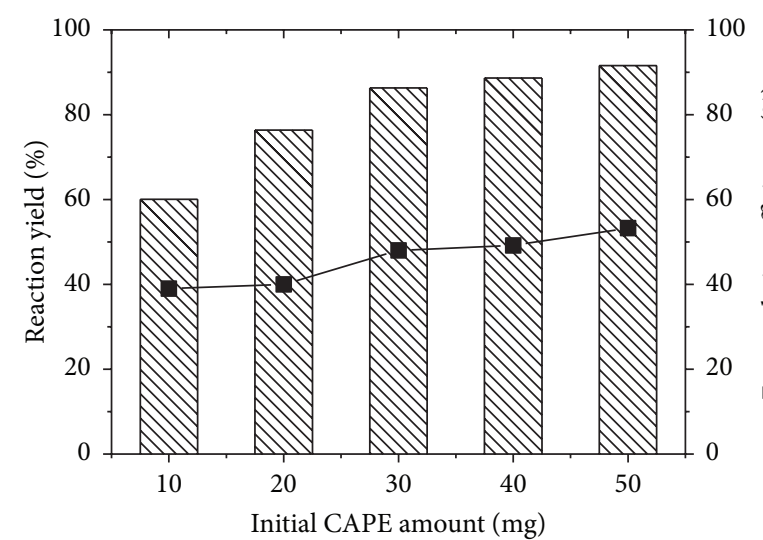

(a)

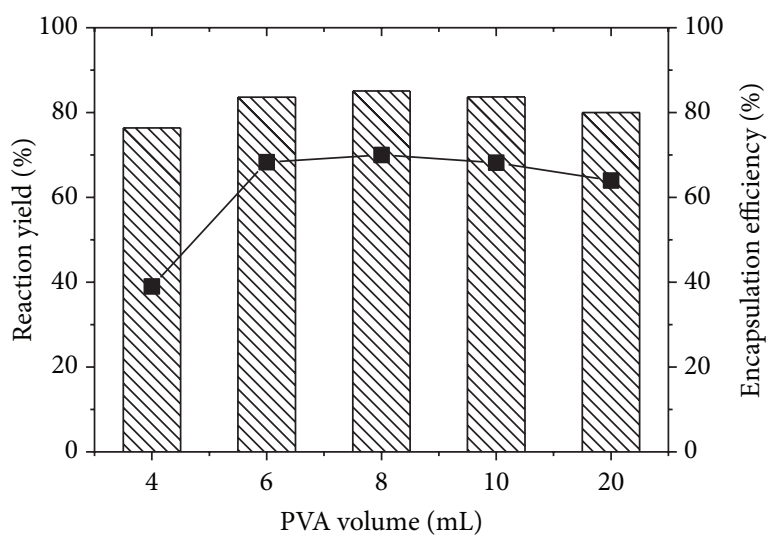

(c)

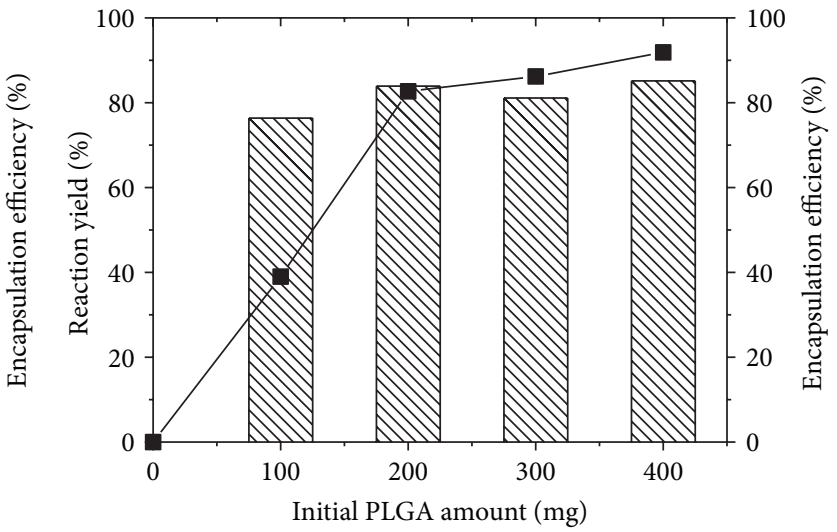

(b)

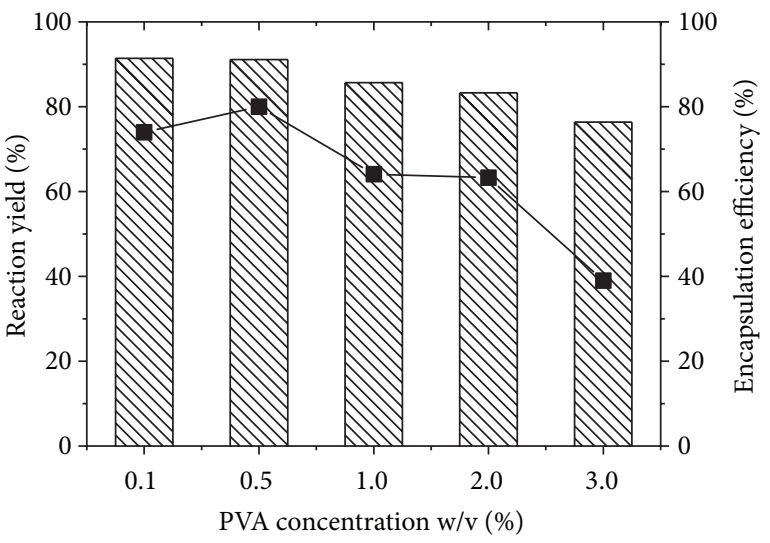

(d)

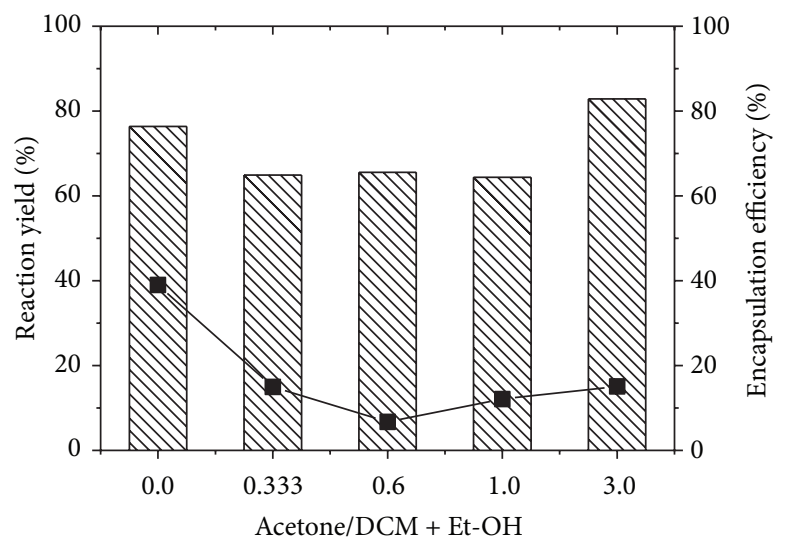

(e)

FIGURE 2: Effect of various process parameters on reaction yield (line) and encapsulation efficiency (column), including the initial CAPE amount (a), initial PLGA amount (b), PVA volume (c), PVA concentration in aqueous phase (d), and solvent type (e) $(n=3)$.

in the yield was confined to $80 \pm 8 \%$ and then systematically decreased with increase in the PVA concentration $(p<0.05)$.

Finally, the effect of solvent type on the reaction yield was shown in Figure 2(e). It can be clearly seen that the reaction yield is significantly affected from the solvent type $(p<0.05)$. The increase in the acetone ratio in solvent mixture led to a decrease the reaction yield. The reaction achieved a minimum yield of $7 \pm 4 \%$ at an acetone: DCM : ethanol ratio of $3: 3: 2$ (for $2 \mathrm{~mL}$ ); then a weak increase $(15 \pm 7)$ was observed in the absence of DCM and the ratio of acetone : ethanol of $3: 1$.

Hence, it can be thought that the reaction yield had a positive relationship with the initial CAPE amount, initial PLGA amount, and PVA volume and a negative relationship with PVA concentration and the presence of acetone in solvent mixture. 
3.2. Effect of Process Parameters on Encapsulation Efficiency. The results of the effect of initial CAPE and PLGA amount, PVA volume and concentration, and solvent type on encapsulation efficiency are listed in Table 2 and illustrated in Figures 2(a) 2(e) respectively.

When a constant initial amount of PLGA (100 mg), PVA volume $(4 \mathrm{~mL})$, PVA concentration $(3 \% \mathrm{w} / \mathrm{v})$, and solvent type (only DCM and ethanol) was maintained, the amount of CAPE varied from 10 to $50 \mathrm{mg}$ (Figure 2(a)). The increase in the initial CAPE amount in organic phase resulted in a significant increase in entrapment efficiency of CAPE $(p<$ $0.05)$, which was in accordance with the results reported $[28,31]$. In the first place the EE remarkably increased (from $60 \pm 3 \%$ to $86 \pm 5 \%$ ) and then reached a plateau when the amount of CAPE was between 30 and $50 \mathrm{mg}$.

It was reported that the drug partition coefficient in internal and external phases significantly affects the encapsulation efficiency of particles using an o/w method [38, 39]. Boury et al. showed that the drug polymer interaction contributes to increasing encapsulation efficiency [40]. Panyam et al. discussed the importance of drug miscibility in polymer for hydrophobic flutamide and reported that higher drug polymer miscibility leads to a higher drug encapsulation [41]. Similar results established by Budhian et al. indicated that the increase in the polymer drug interaction also increases the drug content of particles as a result of high encapsulation efficiency [24].

It was observed in our results that with the increase in initial CAPE amount, the concentration of CAPE in the organic phase increased and more drug molecules could interact with the polymer molecules. This results in the increase in encapsulation efficiency of CAPE.

Figure 2(b) shows the effect of initial PLGA amount on the encapsulation efficiency of CAPE. It is clearly seen that the encapsulation efficiency of CAPE increased significantly $(p<0.05)$ with increase in the PLGA concentration in organic phase. The rise in the initial PLGA amount leads to an increase of organic phase viscosity, which causes more diffusional resistance to drug molecules from organic phase to the aqueous phase [42]. Additionally, increase in the initial PLGA amount resulted in a rise in the particle size. As shown in the literature, larger particles provide higher drug encapsulation efficiency $[24,28,31,43]$. Moreover, due to the increase of particle size the length of diffusional pathway of drugs from organic phase to aqueous phase increases and as a result of this increase the drug loss also decreases. Thereby, reducing the drug loss through diffusion provides increased encapsulation efficiency [42]. Obtained results in accordance with the results reported by Halayqa and Domańska and the authors show that increase in the polymer amount from $0.8 \%$ to $1.6 \%$ increases the encapsulation efficiency $46.6 \%$ to $71.6 \%$ for perphenazine and $44.8 \%$ to $61.6 \%$ for chlorpromazine hydrochloride [31].

The effect of the aqueous phase volume on the encapsulation efficiency of CAPE is shown in Figure 2(c). The encapsulation efficiency of CAPE firstly increased with increase of PVA volume and then reached a plateau that ranged from $76 \pm 4 \%$ to $85 \pm 6 \%$. It was clearly seen from results that encapsulation efficiency is not significantly affected by
PVA volume. Actually, encapsulation efficiency is indirectly affected by aqueous phase volume due to the influence of PVA volume on particle size, which directly affects encapsulation efficiency.

Figure 2(d) shows the effect of PVA concentration on encapsulation efficiency of CAPE. PVA formulated nanoparticles reached maximum encapsulation at low $(0,1 \% \mathrm{w} / \mathrm{v})$ stabilizer concentration. Encapsulation efficiency level reached as high as $91 \pm 5 \%$ and was seen at lowest PVA concentration. When the concentration of stabilizer increased, a linear and small reduction in overall CAPE encapsulation was seen (Table 2). Obtained results are in agreement with literature [28, 34, 43, 44]. Cooper and Harirforoosh synthesized diclofenac encapsulated PLGA nanoparticles with polyvinylalcohol and didodecyldimethylammonium bromide (DMAB) as a stabilizer. These group observed that encapsulation of diclofenac decreases with increase in the concentration of each of the two stabilizers [44].

The effect of the solvent type (acetone to DCM + ethanol volume ratio is in the range of 0 to 3.0) on encapsulation efficiency of CAPE was shown in Figure 2(e). It was seen in Figure 2(e) and Table 2 that encapsulation efficiency is significantly decreased by addition of acetone to organic solvent mixture and reached a plateau with the rise of acetone volume ratio (from 0.333 to 1.0 ) and then increase for acetone to DCM + ethanol volume ratio was equal to 3.0. This occurred possibly because of the change of Acetone/DCM + ethanol volume ratio affecting the dispersion of CAPE in organic phase. Encapsulation efficiency reached maximum for acetone/DCM + ethanol volume ratio being 3.0; however in this condition the significant reduction in reaction yield $(15 \pm 7 \%)$ was observed.

\subsection{Effect of Process Parameters on Particle Size and Polydis-} persity Index. In this study the effects of initial CAPE and PLGA amount, PVA concentration, PVA volume (aqueousto-organic phase volume ratio), and solvent type on mean particle size of nanoparticles were investigated. The particle size values for all batches show a wide variation in response that ranged from a minimum of $158 \pm 9 \mathrm{~nm}$ to a maximum of $469 \pm 15 \mathrm{~nm}$. Obtained results openly indicate that the mean particle size is strongly related on selected process parameters.

The effect of initial CAPE amount on nanoparticles size was shown in Figure 3(a). Initial CAPE amount in organic phase had no significant effect on the mean particle size which was similar to many earlier studies with hydrophobic molecules $[24,28]$.

Figure 3(b) shows that the mean particle size of CAPE loaded PLGA nanoparticles increased significantly with the increase of initial PLGA concentration. It can be observed that increase of the PLGA amount from $100 \mathrm{mg}$ to $400 \mathrm{mg}$ increases the mean particle size from $208 \pm 8 \mathrm{~nm}$ to $437 \pm$ $9 \mathrm{~nm}$. The same results were also previously reported by other researcher [28, 31, 45-48]. This could explain that increase of the PLGA amount in organic phase leads to rise of solution viscosity and decrease of net shear stress. As a result of reducing shear stress larger particles are formed. Additionally, increase in the viscosity could prevent quick dispersion 


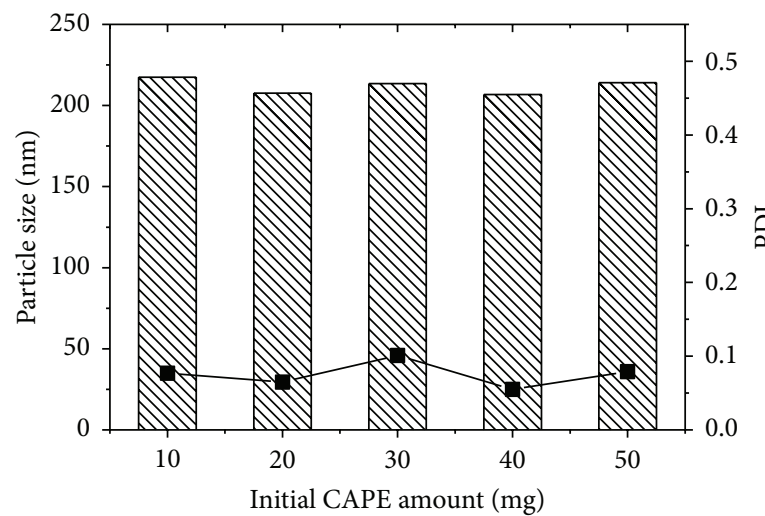

(a)

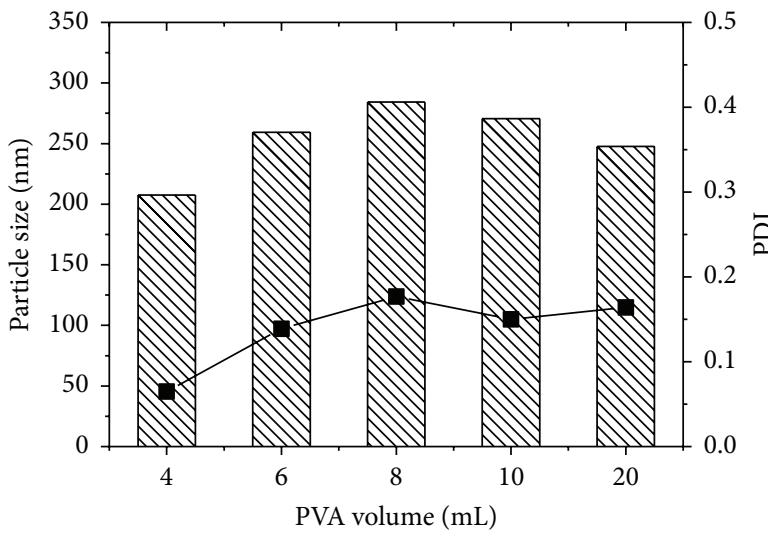

(c)

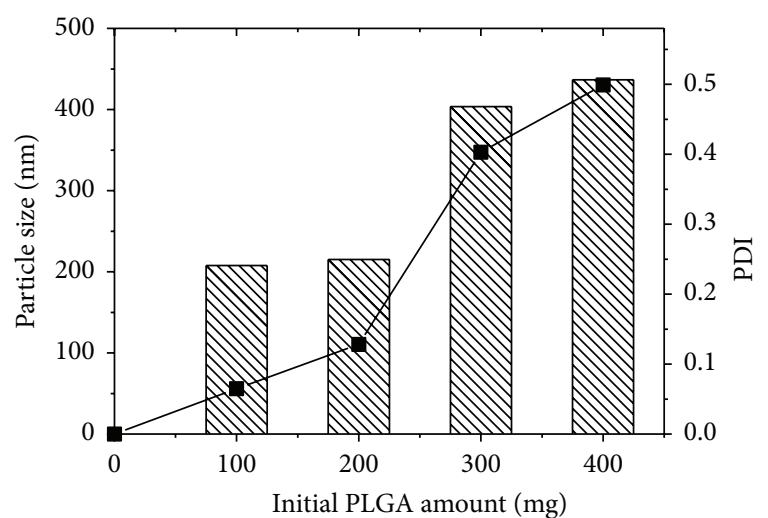

(b)

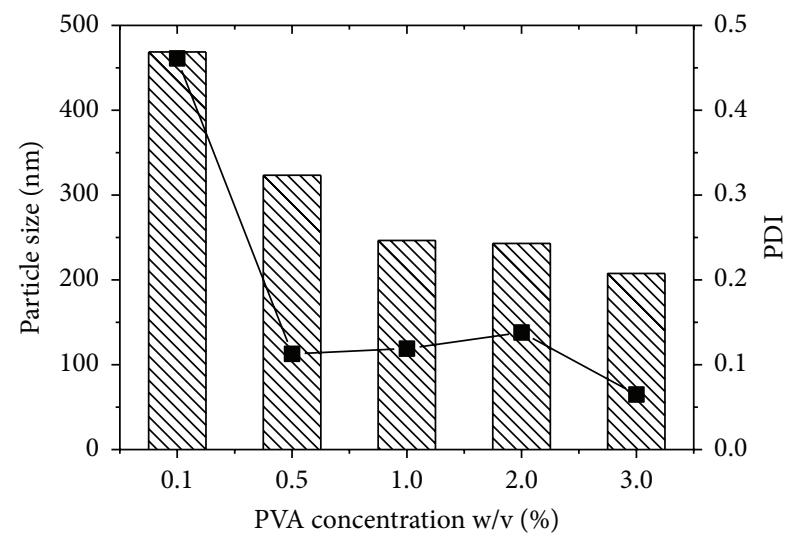

(d)

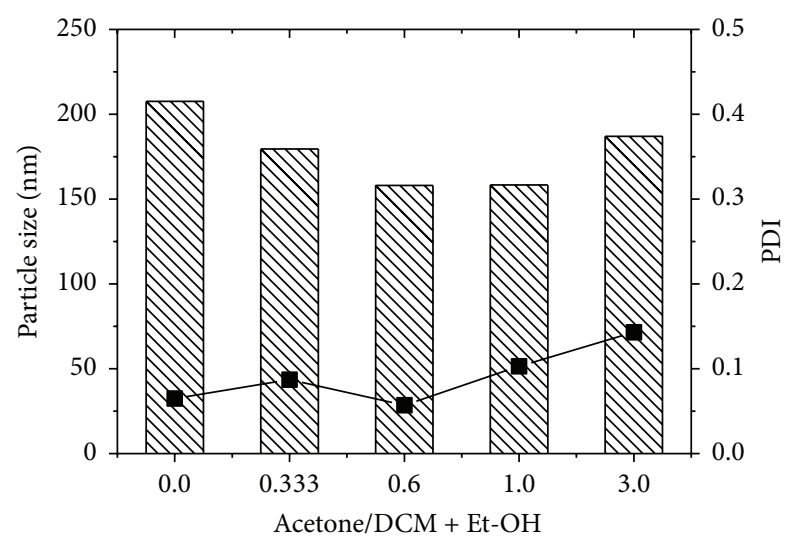

(e)

FIGURE 3: Effect of various process parameters on particle size (column) and PDI (line), including the initial CAPE amount (a), initial PLGA amount (b), PVA volume (c), PVA concentration in aqueous phase (d), and solvent type (e) $(n=3)$.

of polymer solution into the aqueous PVA phase, resulting in bigger droplets which formed larger nanoparticles after evaporation of oil phase.

Figure 3(c) shows that the mean particle size dependence to PVA volume (aqueous-to-organic phase volume ratio) in aqueous phase for CAPE loaded nanoparticles. It can be seen in the figure that the mean particle size was first increased and then decreased with increase in the PVA volume. Increase in PVA volume causes an increase in the PVA amount, resulting in interfacial tension reduction thence decreasing the mean particle size [28]. Furthermore, the increased the total system volume would reduce the net shear stress because of a constant energy source causing to formation of larger particles $[28,47]$.

In this study PVA was applied as emulsifier in the aqueous phase $(\mathrm{w})$ to simplify the preparation of $\mathrm{o} / \mathrm{w}$ single emulsion. Figure 3(d) shows the effect of PVA concentration in aqueous phase on the mean particles size. Different PVA concentrations are selected while keeping the other system parameters constant and PVA was studied between $0.1 \%$ 
and $3.0 \%(\mathrm{w} / \mathrm{v})$ concentrations. It can be observed that the mean particle size first decreased significantly $(p<0.05)$ with increase of PVA concentration up to $1 \%(\mathrm{w} / \mathrm{v})$ and then gradually decreased from $246 \pm 12$ to $208 \pm 8 \mathrm{~nm}(3 \% \mathrm{w} / \mathrm{v})$. This can explain that PVA play remarkable role in reducing the interfacial tension and in this way disperse the emulsion nanodroplets and prevent them from agglomeration [31, 49]. It was reported that increase in the PVA concentration may lead to the decrease of particle size due to tight surface that was formed from PVA macromolecular chains in high surfactant concentrations [31, 43, 45, 50, 51]. Otherwise the viscosity of the system increases with the increase of the PVA concentration; this leads to decrease of net share stress which results in formation of larger molecules [24]. Conversely, in our results the decreases in the mean particles size reached a plateau after the concentration of PVA is between 1 and $3 \% \mathrm{w} / \mathrm{v}$. The same results observed Budhian and colleagues study which show that the size of Haloperidol loaded PLGA nanoparticles first decreases (up to $1 \% \mathrm{w} / \mathrm{v}$ ), then reached a plateau (up to $5 \% \mathrm{w} / \mathrm{v}$ ), and gradually increases (higher concentration than $5 \% \mathrm{w} / \mathrm{v}$ ) by increasing of PVA concentration for sonication methods [24]. According to our results it can be conclude that increase in the PVA concentration more than $3 \%(\mathrm{w} / \mathrm{v})$ may result in an increase of the mean particle size of nanoparticles.

The solvent type also has a remarkable effect on the mean particle size. Figure 3(e) shows the significant $(p<$ 0.05 ) reduction of mean particle size with acetone-to-DCM + ethanol ratio. Addition of water-miscible organic solvent leads to significant decrease of interfacial tension because of rapid dispersion of acetone into the external aqueous phase, thereby decreasing the particle size which was consistent with literatures $[28,43,52]$.

The PDI is an important property of particles and refers to broadness of a mean particle size distribution for dispersion of nanoparticle [53]. Therefore, effect of process parameters on PDI values of nanoparticles was examined in this study. The positive and negative effect of process parameters on PDI of nanoparticles can be seen in Table 2 and Figures 3(a) 3(e) respectively.

Generally, PDI $<0.3$ is considered a requested value for an acceptable narrow range of nanoparticle size [54]. In the first place particles prepared by increasing amounts of CAPE (10 mg to $50 \mathrm{mg}$ ) were narrowly distributed and had the lowest PDI values (varied between $0.055 \pm 0.005$ and $0.101 \pm 0.008$ ) (Figure 3(a)).

Obtained results showed that the PLGA concentration is the most important factor affecting the PDI of nanoparticles. The PDI values varied between $0.065 \pm 0.006$ and $0.499 \pm 0.066$ for $100 \mathrm{mg}$ to $400 \mathrm{mg}$ PLGA amount, respectively. From the results it can be seen that almost monodisperse nanoparticles were produced for $100 \mathrm{mg}$ polymer amount and the mean particle size distribution was broadened with increase of the initial PLGA amount (Figure 3(b)).

From Figure 3(c) and Table 2, it can be clearly seen that all of PVA volume was sufficient for reducing PDI below 0.3. In this group PDI value of particles first increased from $0.065 \pm 0.006$ to $0.177 \pm 0.005$ (for $8 \mathrm{~mL}$ of PVA) and then slowly decreased by increase in the aqueous phase volume.
Also, PVA concentration generally exhibited a negative influence on PDI (Figure 3(d)). It was observed in our results that the polydispersity index decreased when PVA concentration increases from $0.1 \%$ to $3 \%$.

Figure 3(e) shows the effect of solvent type on PDI value of CAPE loaded nanoparticles. It was seen in the figure that PDI is affected from addition of acetone in solvent mixture especially in the lack of DCM. In the absence of acetone (DCM to ethanol ratio of $3: 1$ ) the PDI value of nanoparticles was obtained $0.065 \pm 0.006$, addition of acetone increased PDI up to $0.103 \pm 0.008$, and this value reached maximum $(0.143 \pm 0.014)$ for acetone to DCM + ethanol ratio equal to 3.0 .

3.4. Effect of Process Parameters on Zeta Potential. Zeta potential is another important physicochemical parameter in nanoparticles that influences stability of nanoparticle suspension. Extremely negative or positive zeta potential causes high repulsive forces and prevents agglomeration of nanoparticles [55]. Under these conditions long-term stability of the nanoparticles can be anticipated [55]. It can be seen in Table 2 that the zeta potential values of all nanoparticles were negative and ranged between $-12.7 \pm 1.3 \mathrm{mV}$ and $-22.4 \pm$ $0.7 \mathrm{mV}$. This expected negativity was based on presence of ionized carboxyl groups [51] and PVA [55] on the surface of the nanoparticles. Also, higher negative zeta potential can enhance the dispersion of the nanoparticles in physiological systems and water [54].

3.5. Optimization of CAPE Loaded PLGA Nanoparticles. Obtained results show that CAPE was successfully encapsulated into the PLGA nanoparticles. On the other hand, according to our criteria for lower particle size and higher entrapment efficiency, we prepared CAPE loaded PLGA nanoparticles as follows: $50 \mathrm{mg}$ of CAPE and $100 \mathrm{mg}$ of PLGA were dissolved into $0.5 \mathrm{~mL}$ of ethanol and $1.5 \mathrm{~mL}$ of DCM, respectively. The mixed organic phase was emulsified with $6 \mathrm{~mL}$ of aqueous PVA solution $(2 \% \mathrm{w} / \mathrm{v})$ by sonication (output power $70 \mathrm{~W}$, power of $80 \%$, and 2 minutes) using a microtip probe sonicator in ice bath. The emulsion was stirred overnight for evaporation of organic phase. The resulting particles were collected and lyophilized.

\subsection{Detailed Characterization of Optimized Nanoparticles.} For the detailed characterization of optimized nanoparticles firstly, we determined reaction yield, encapsulation efficiency, and loading capacity. Lyophilized nanoparticles were weighed and yield of the process was calculated to be $70 \pm$ $6 \%$. The corresponding encapsulation efficiency and loading capacity of optimized nanoparticles were calculated $89 \pm 3$ and $42 \pm 4$, respectively.

3.6.1. DLS and ELS Analysis. Dynamic and electrophoretic light scattering techniques were used for particle size and zeta potential measurement of nanoparticles. Figure 4 depicts the formation of nearly monodisperse CAPE nanoparticle with the mean particle size being $163 \pm 2 \mathrm{~nm}(\mathrm{PDI}=0.119 \pm 0.002)$. The zeta potential of CAPE loaded nanoparticles was equal to $-34.4 \pm 2.5$ (Figure 4$)$. 


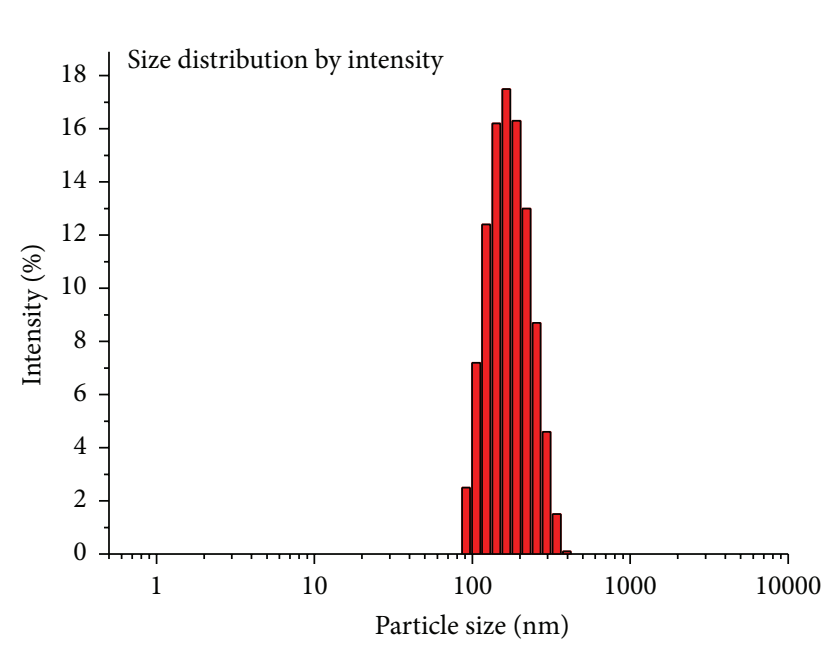

(a)

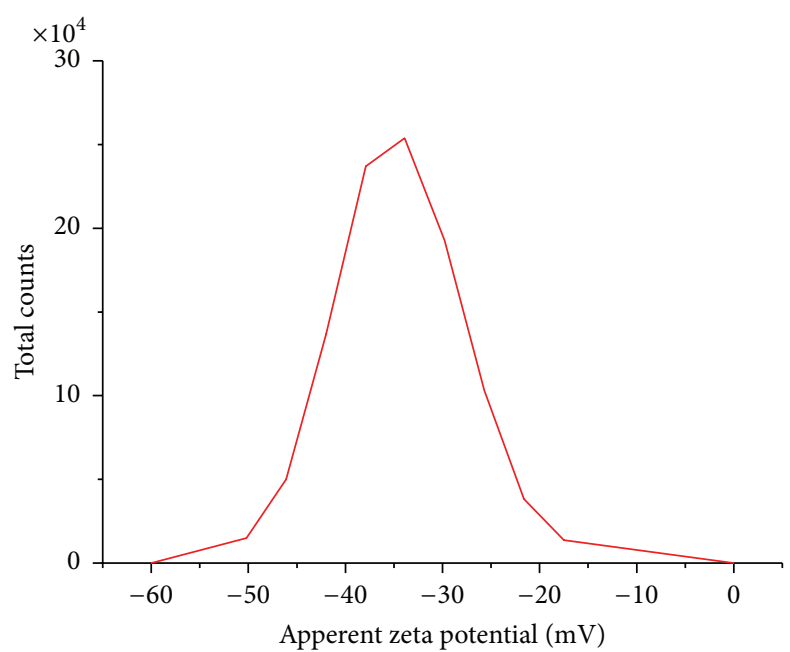

(b)

FIGURE 4: Particle size (a) and zeta potential (b) distribution of optimized nanoparticle.

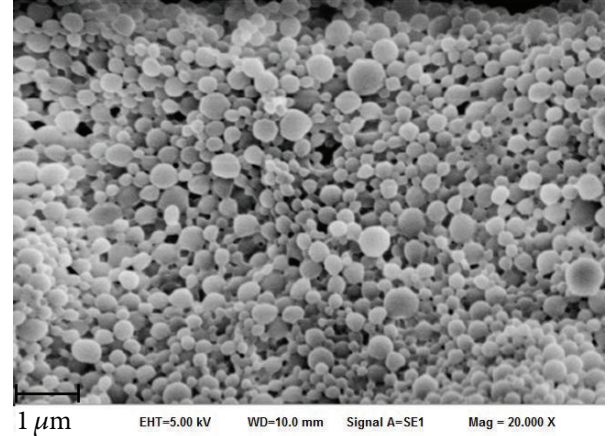

FiguRE 5: Scanning electron microscopy image of optimized nanoparticles.

3.6.2. SEM Analysis. The surface morphology of the optimized nanoparticle was determined by SEM. As shown in Figure 5, smooth and spherical shape nanoparticles with uniform distribution were obtained. SEM results are also in agreement with DLS results that the particles optimized nanoparticles have a uniform size distribution and low polydispersity index.

3.6.3. FTIR Analysis. Figure 6 shows FTIR spectra of PLGA (a), optimized nanoparticle (b), and pure CAPE (c). In the FTIR spectrum of PLGA and nanoparticles the bands at $2993 \mathrm{~cm}^{-1}$ and $2989 \mathrm{~cm}^{-1}$ were $\mathrm{C}-\mathrm{H}$ stretch of $\mathrm{CH}_{2}$ and $\mathrm{C}-\mathrm{H}$ stretch of $-\mathrm{C}-\mathrm{H}-$, respectively.

A band at $1751 \mathrm{~cm}^{-1}$ was assigned to the stretching vibration of $\mathrm{C}=\mathrm{O}$ of ester bond (strong and narrow) and 1165$1087 \mathrm{~cm}^{-1}$ was attributed to $\mathrm{C}-\mathrm{O}$ stretching, which belongs to the characteristic peaks of PLGA molecule [56]. It can be seen from the FT-IR spectrum of free CAPE that the bands obtained at $3471 \mathrm{~cm}^{-1}$ and $3320 \mathrm{~cm}^{-1}$ were assigned to $-\mathrm{OH}$ stretching. The strong and narrow peaks at $1681 \mathrm{~cm}^{-1}$, $1600 \mathrm{~cm}^{-1}$, and $1172 \mathrm{~cm}^{-1}$ were also attributed $\mathrm{C}=\mathrm{O}, \mathrm{C}=\mathrm{C}$, and

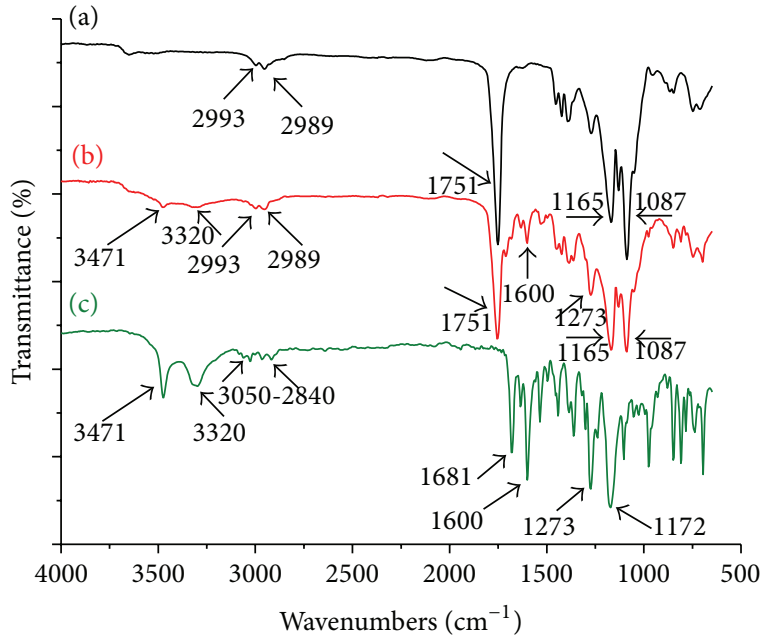

FIGURE 6: FTIR spectrum of PLGA (a), optimized nanoparticle (b), and free CAPE (c).

C-O stretching, respectively. However, in the FTIR spectra of CAPE loaded nanoparticles, the major peaks of CAPE at $3471 \mathrm{~cm}^{-1}, 3320 \mathrm{~cm}^{-1}$, and $1600 \mathrm{~cm}^{-1}$ were significantly decreased and the presence of these characteristic peaks is a confirmation of CAPE encapsulation on PLGA nanoparticles successfully.

3.6.4. Release Study. Figure 7 illustrates in vitro release profiles of CAPE from nanoparticles prepared at different process parameters. The nanoparticles showed a typical three-phase release pattern in $\mathrm{pH} 7.4$.

For all the nanoparticles prepared with different process parameters, an initial burst release, caused by diffusion of drug, continuing for $24 \mathrm{~h}$ of incubation was observed. On the other hand, as seen in Figure 7 depending on the process parameters, the percentage of burst release varies between 


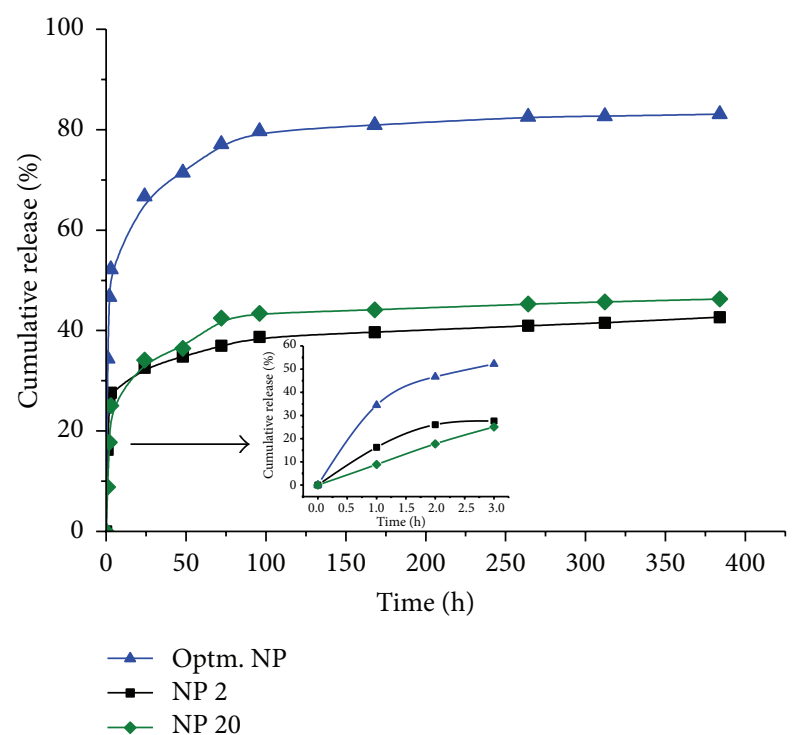

FIGURE 7: In vitro release profile of CAPE in $\mathrm{pH} 7.4$ phosphate buffer from CAPE loaded PLGA nanoparticles corresponding to different polymer/drug ratios.

$32.59 \%$ and $66.72 \%$. In the second phase of release, caused by drug diffusion and polymer chain cleavage, approximately constant and low level of CAPE release rate was observed. In the last step of CAPE release, continuous release almost close to linear was observed over 7-16 days and reached $42.65 \%$, $46.26 \%$, and $83.08 \%$ for NP 2, NP 20, and optimized NP, respectively, at the end of 16 th day.

In Figure 7 it was clearly seen that when the polymer/drug ratio decreased the burst release of CAPE increases. Usually initial burst release occurred due to the fast diffusion of drug absorbed on the surface or localized near surface of particles [57]. In the low polymer/drug ratio, PLGA was not enough to encapsulate the drug; thus most of the drug was adsorbed on or localized near the surface of nanoparticles, which resulted in higher burst release [57]. The minimum burst release was observed for NP 20 (polymer/drug ratio equal to 0.05) and the highest was optimized NP (polymer/drug ratio equal to $0.5)$. In accordance with these results the initial burst release of NP 2 (drug/polymer ratio equal to 0.2 ) higher than NP 20 and lower than optimized NP was obtained.

\section{Conclusion}

CAPE has numerous biological and pharmacological effects, such as antiviral, antioxidant, and anticancer activities. Although CAPE has many biological properties, its usage in pharmaceutical area is limited due to its low aqueous solubility and instability in biological system. One of the methods to overcome these problems is to encapsulation of the CAPE into biodegradable nanoparticular system.

Poly(DL, lactic-co-glycolic acid) is the most frequently used biodegradable, biocompatible copolymer for developing nanoparticles in controlled release (CR) applications. PLGA nanoparticles are especially beneficial in drug delivery system for low-water soluble compounds. The small particle size and controlled release properties of nanoparticles can improve the water solubility, bioavailability, biocompatibility, and absorption of the delivered bioactive molecules.

Thus, in this study, for the first time caffeic acid phenethyl ester loaded PLGA nanoparticles were successfully produced by a single emulsion solvent evaporation method. Additionally, the influences of different process parameters on reaction yield, particle size, encapsulation efficiency, polydispersity index, and zeta potential of nanoparticles were systematically investigated and optimal process parameters were determined. Particularly, the initial CAPE and PLGA amount, PVA concentration, and solvent type were found to have played overpowering role in encapsulation efficiency and particle size.

The final optimal value for the initial CAPE and PLGA amount, PVA volume, and concentration were determined at $50 \mathrm{mg}, 100 \mathrm{mg}, 6 \mathrm{~mL}$, and $2 \% \mathrm{w} / \mathrm{v}$, respectively. A particle size equal to $163 \pm 2 \mathrm{~nm}$ with low polydispersity index $0.119 \pm$ $0.002,-34.4 \pm 2.5 \mathrm{mV}$ zeta potential, and percentage encapsulation of $89 \pm 3 \%$ was achieved under the optimal condition employed. Interestingly, the optimized nanoparticles showed a high (83.08\%) and sustained CAPE release (lasting for 16 days) compared to nonoptimized nanoparticle.

In conclusion, high encapsulation efficiency with small size and sustained release make CAPE loaded nanoparticles a suitable candidate for the further development of nanomedicine. Additionally studies are currently in progress to test the antimicrobial activity of CAPE loaded PLGA nanoparticle against different Gram positive and Gram negative bacteria.

\section{Conflict of Interests}

The author declares that there is no conflict of interests regarding the publication of this paper.

\section{Acknowledgment}

This research has been supported by Yildiz Technical University Scientific Research Projects Coordination Department, Project no. 2014-07-04-GEP02.

\section{References}

[1] H.-Y. Lee, Y.-I. Jeong, E. J. Kim et al., "Preparation of caffeic acid phenethyl ester-incorporated nanoparticles and their biological activity," Journal of Pharmaceutical Sciences, vol. 104, no. 1, pp. 144-154, 2015.

[2] M. R. Fesen, Y. Pommier, F. Leteurtre, S. Hiroguchi, J. Yung, and K. W. Kohn, "Inhibition of HIV-1 integrase by flavones, caffeic acid phenethyl ester (CAPE) and related compounds," Biochemical Pharmacology, vol. 48, no. 3, pp. 595-608, 1994.

[3] G. F. Sud'Ina, O. K. Mirzoeva, M. A. Pushkareva, G. A. Korshunova, N. V. Sumbatyan, and S. D. Varfolomeev, "Caffeic acid phenethyl ester as a lipoxygenase inhibitor with antioxidant properties," FEBS Letters, vol. 329, no. 1-2, pp. 21-24, 1993.

[4] S.-G. Park, D.-Y. Lee, S.-K. Seo et al., "Evaluation of anti-allergic properties of caffeic acid phenethyl ester in a murine model 
of systemic anaphylaxis," Toxicology and Applied Pharmacology, vol. 226, no. 1, pp. 22-29, 2008.

[5] Y.-J. Chen, M.-S. Shiao, and S.-Y. Wang, “The antioxidant caffeic acid phenethyl ester induces apoptosis associated with selective scavenging of hydrogen peroxide in human leukemic HL-60 cells," Anti-Cancer Drugs, vol. 12, no. 2, pp. 143-149, 2001.

[6] P. Michaluart, J. L. Masferrer, A. M. Carothers et al., "Inhibitory effects of caffeic acid phenethyl ester on the activity and expression of cyclooxygenase- 2 in human oral epithelial cells and in a rat model of inflammation," Cancer Research, vol. 59, no. 10, pp. 2347-2352, 1999.

[7] M. Kartal, S. Yildiz, S. Kaya, S. Kurucu, and G. Topçu, "Antimicrobial activity of propolis samples from two different regions of Anatolia," Journal of Ethnopharmacology, vol. 86, no. 1, pp. 69-73, 2003.

[8] J. H. Park, J. K. Lee, H. S. Kim et al., "Immunomodulatory effect of caffeic acid phenethyl ester in Balb/c mice," International Immunopharmacology, vol. 4, no. 3, pp. 429-436, 2004.

[9] J. Wu, C. Omene, J. Karkoszka et al., "Caffeic acid phenethyl ester (CAPE), derived from a honeybee product propolis, exhibits a diversity of anti-tumor effects in pre-clinical models of human breast cancer," Cancer Letters, vol. 308, no. 1, pp. 4353,2011 .

[10] H.-F. Liao, Y.-Y. Chen, J.-J. Liu et al., "Inhibitory effect of caffeic acid phenethyl ester on angiogenesis, tumor invasion, and metastasis," Journal of Agricultural and Food Chemistry, vol. 51, no. 27, pp. 7907-7912, 2003.

[11] A. Ilhan, U. Koltuksuz, S. Ozen, E. Uz, H. Ciralik, and O. Akyol, "The effects of caffeic acid phenethyl ester (CAPE) on spinal cord ischemia/reperfusion injury in rabbits," European Journal of Cardio-Thoracic Surgery, vol. 16, no. 4, pp. 458-463, 1999.

[12] T. R. Burke Jr., M. R. Fesen, A. Mazumder et al., "Hydroxylated aromatic inhibitors of HIV-1 integrase," Journal of Medicinal Chemistry, vol. 38, no. 21, pp. 4171-4178, 1995.

[13] M. Watabe, K. Hishikawa, A. Takayanagi, N. Shimizu, and T. Nakaki, "Caffeic acid phenethyl ester induces apoptosis by inhibition of $\mathrm{NF} \kappa \mathrm{B}$ and activation of Fas in human breast cancer MCF-7 cells," Journal of Biological Chemistry, vol. 279, no. 7, pp. 6017-6026, 2004.

[14] K. Choi, Y.-H. Han, and C. Choi, " $N$-acetyl cysteine and caffeic acid phenethyl ester sensitize astrocytoma cells to Fas-mediated cell death in a redox-dependent manner," Cancer Letters, vol. 257, no. 1, pp. 79-86, 2007.

[15] N. Berger, H. Ben Bassat, B. Y. Klein, and R. Laskov, "Cytotoxicity of NF- $\kappa$ B inhibitors Bay 11-7085 and caffeic acid phenethyl ester to Ramos and other human B-lymphoma cell lines," Experimental Hematology, vol. 35, no. 10, pp. 1495-1509, 2007.

[16] K. McEleny, R. Coffey, C. Morrissey, J. M. Fitzpatrick, and R. W. G. Watson, "Caffeic acid phenethyl ester-induced PC-3 cell apoptosis is caspase-dependent and mediated through the loss of inhibitors of apoptosis proteins," BJU International, vol. 94, no. 3, pp. 402-406, 2004.

[17] U.-H. Jin, K.-H. Song, M. Motomura et al., "Caffeic acid phenethyl ester induces mitochondria-mediated apoptosis in human myeloid leukemia U937 cells," Molecular and Cellular Biochemistry, vol. 310, no. 1-2, pp. 43-48, 2008.

[18] T. Nagaoka, A. H. Banskota, Y. Tezuka et al., "Inhibitory effects of caffeic acid phenethyl ester analogues on experimental lung metastasis of murine colon 26-L5 carcinoma cells," Biological and Pharmaceutical Bulletin, vol. 26, no. 5, pp. 638-641, 2003.
[19] S. Gao and M. Hu, "Bioavailability challenges associated with development of anti-cancer phenolics," Mini-Reviews in Medicinal Chemistry, vol. 10, no. 6, pp. 550-567, 2010.

[20] E. Kerns and L. Di, Drug-Like Properties: Concepts, Structure Design and Methods: From ADME to Toxicity Optimization, Academic Press, 2010.

[21] Y.-C. Weng, S.-T. Chuang, Y.-C. Lin et al., "Caffeic acid phenylethyl amide protects against the metabolic consequences in diabetes mellitus induced by diet and streptozocin," EvidenceBased Complementary and Alternative Medicine, vol. 2012, Article ID 984780, 12 pages, 2012.

[22] X. Wang, J. Pang, J. A. Maffucci et al., "Pharmacokinetics of caffeic acid phenethyl ester and its catechol-ring fluorinated derivative following intravenous administration to rats," Biopharmaceutics \& Drug Disposition, vol. 30, no. 5, pp. 221-228, 2009.

[23] A. Kumari, S. K. Yadav, and S. C. Yadav, "Biodegradable polymeric nanoparticles based drug delivery systems," Colloids and Surfaces B: Biointerfaces, vol. 75, no. 1, pp. 1-18, 2010.

[24] A. Budhian, S. J. Siegel, and K. I. Winey, "Haloperidol-loaded PLGA nanoparticles: systematic study of particle size and drug content," International Journal of Pharmaceutics, vol. 336, no. 2, pp. 367-375, 2007.

[25] D. K. Sahana, G. Mittal, V. Bhardwaj, and M. N. V. R. Kumar, "PLGA nanoparticles for oral delivery of hydrophobic drugs: Influence of organic solvent on nanoparticle formation and release behavior in vitro and in vivo using estradiol as a model drug," Journal of Pharmaceutical Sciences, vol. 97, no. 4, pp. 1530-1542, 2008.

[26] B. N. Singh, S. Shankar, and R. K. Srivastava, "Green tea catechin, epigallocatechin-3-gallate (EGCG): mechanisms, perspectives and clinical applications," Biochemical Pharmacology, vol. 82, no. 12, pp. 1807-1821, 2011.

[27] T. Chaowanachan, E. Krogstad, C. Ball, and K. A. Woodrow, "Drug synergy of tenofovir and nanoparticle-based antiretrovirals for HIV prophylaxis," PLoS ONE, vol. 8, no. 4, Article ID e61416, 2013.

[28] X. Song, Y. Zhao, S. Hou et al., "Dual agents loaded PLGA nanoparticles: systematic study of particle size and drug entrapment efficiency," European Journal of Pharmaceutics and Biopharmaceutics, vol. 69, no. 2, pp. 445-453, 2008.

[29] A. S. Hussein, N. Abdullah, and A. Fakru'l-Razi, “Optimizing the process parameters for encapsulation of linamarin into PLGA nanoparticles using double emulsion solvent evaporation technique," Advances in Polymer Technology, vol. 32, supplement 1, pp. E486-E504, 2013.

[30] S. Derman and Z. M. Akdeste, "Particle size and zeta potential investigation of synthetic peptide-protein conjugates," Turkish Journal of Biochemistry, vol. 40, no. 4, pp. 282-289, 2015.

[31] M. Halayqa and U. Domańska, "PLGA biodegradable nanoparticles containing perphenazine or chlorpromazine hydrochloride: effect of formulation and release," International Journal of Molecular Sciences, vol. 15, no. 12, pp. 23909-23923, 2014.

[32] S. Dixit, S. R. Singh, A. N. Yilma, R. D. Agee, M. Taha, and V. A. Dennis, "Poly(lactic acid)—poly(ethylene glycol) nanoparticles provide sustained delivery of a Chlamydia trachomatis recombinant MOMP peptide and potentiate systemic adaptive immune responses in mice," Nanomedicine: Nanotechnology, Biology, and Medicine, vol. 10, no. 6, pp. 1311-1321, 2014.

[33] E. Clelikkaya, E. B. DenkbasL, and E. PisLkin, "Rifampicin carrying poly (D, L-Lactide)/Poly(Ethylene Glycol) microspheres: 
loading and release," Artificial Organs, vol. 20, no. 7, pp. 743-751, 1996.

[34] F. Esmaeili, M. Hosseini-Nasr, M. Rad-Malekshahi, N. Samadi, F. Atyabi, and R. Dinarvand, "Preparation and antibacterial activity evaluation of rifampicin-loaded poly lactide-coglycolide nanoparticles," Nanomedicine: Nanotechnology, Biology, and Medicine, vol. 3, no. 2, pp. 161-167, 2007.

[35] SPSS, SPSS for Windows Version 15.0, SPSS, Chicago, Ill, USA, 2006.

[36] S. Mao, J. Xu, C. Cai, O. Germershaus, A. Schaper, and T. Kissel, "Effect of WOW process parameters on morphology and burst release of FITC-dextran loaded PLGA microspheres," International Journal of Pharmaceutics, vol. 334, no. 1-2, pp. 137148, 2007.

[37] S. Mao, Y. Shi, L. Li, J. Xu, A. Schaper, and T. Kissel, "Effects of process and formulation parameters on characteristics and internal morphology of poly(d,l-lactide-co-glycolide) microspheres formed by the solvent evaporation method," European Journal of Pharmaceutics and Biopharmaceutics, vol. 68, no. 2, pp. 214-223, 2008.

[38] R. Bodmeier and J. W. McGinity, "Polylactic acid microspheres containing quinidine base and quinidine sulphate prepared by the solvent evaporation technique. I. Methods and morphology," Journal of Microencapsulation, vol. 4, no. 4, pp. 279-288, 1987.

[39] A. Al-Maaieh and D. R. Flanagan, "Salt and cosolvent effects on ionic drug loading into microspheres using an O/W method," Journal of Controlled Release, vol. 70, no. 1-2, pp. 169-181, 2001.

[40] F. Boury, H. Marchais, J. E. Proust, and J. P. Benoit, "Bovine serum albumin release from poly $(\alpha$-hydroxy acid $)$ microspheres: effects of polymer molecular weight and surface properties," Journal of Controlled Release, vol. 45, no. 1, pp. 75-86, 1997.

[41] J. Panyam, D. William, A. Dash, D. Leslie-Pelecky, and V. Labhasetwar, "Solid-state solubility influences encapsulation and release of hydrophobic drugs from PLGA/PLA nanoparticles," Journal of Pharmaceutical Sciences, vol. 93, no. 7, pp. 1804-1814, 2004.

[42] A. Kumar, H. M. Mansour, A. Friedman, and E. R. Blough, Nanomedicine in Drug Delivery, CRC Press, New York, NY, USA, 2013.

[43] X. Song, Y. Zhao, W. Wu et al., "PLGA nanoparticles simultaneously loaded with vincristine sulfate and verapamil hydrochloride: systematic study of particle size and drug entrapment efficiency," International Journal of Pharmaceutics, vol. 350, no. 1-2, pp. 320-329, 2008.

[44] D. L. Cooper and S. Harirforoosh, "Design and optimization of PLGA-based diclofenac loaded nanoparticles," PLoS ONE, vol. 9, no. 1, Article ID e87326, 2014.

[45] H.-Y. Kwon, J.-Y. Lee, S.-W. Choi, Y. Jang, and J.-H. Kim, "Preparation of PLGA nanoparticles containing estrogen by emulsification-diffusion method," Colloids and Surfaces A: Physicochemical and Engineering Aspects, vol. 182, no. 1-3, pp. 123-130, 2001.

[46] M. Chorny, I. Fishbein, H. D. Danenberg, and G. Golomb, "Lipophilic drug loaded nanospheres prepared by nanoprecipitation: effect of formulation variables on size, drug recovery and release kinetics," Journal of Controlled Release, vol. 83, no. 3, pp. 389-400, 2002.

[47] R. M. Mainardes and R. C. Evangelista, "PLGA nanoparticles containing praziquantel: effect of formulation variables on size distribution," International Journal of Pharmaceutics, vol. 290, no. 1-2, pp. 137-144, 2005.

[48] B. Wang, G. Chen, Z. Mao, Y. Zhang, D. Yu, and C. Gao, "Preparation and cellular uptake of PLGA particles loaded with lamivudine," Chinese Science Bulletin, vol. 57, no. 31, pp. 39853993, 2012.

[49] S. Galindo-Rodriguez, E. Allémann, H. Fessi, and E. Doelker, "Physicochemical parameters associated with nanoparticle formation in the salting-out, emulsification-diffusion, and nanoprecipitation methods," Pharmaceutical Research, vol. 21, no. 8, pp. 1428-1439, 2004.

[50] A. P. Ranjan, A. Mukerjee, L. Helson, and J. K. Vishwanatha, "Scale up, optimization and stability analysis of Curcumin C3 complex-loaded nanoparticles for cancer therapy," Journal of Nanobiotechnology, vol. 10, article 38, 2012.

[51] S. K. Sahoo, J. Panyam, S. Prabha, and V. Labhasetwar, "Residual polyvinyl alcohol associated with poly (D,L-lactide-coglycolide) nanoparticles affects their physical properties and cellular uptake," Journal of Controlled Release, vol. 82, no. 1, pp. 105-114, 2002.

[52] T. Niwa, H. Takeuchi, T. Hino, N. Kunou, and Y. Kawashima, "In vitro drug release behavior of D,L-lactide/glycolide copolymer (PLGA) nanospheres with nafarelin acetate prepared by a novel spontaneous emulsification solvent diffusion method," Journal of Pharmaceutical Sciences, vol. 83, no. 5, pp. 727-732, 1994.

[53] R. Dangi and S. Shakya, "Preparation, optimization and characterization of PLGA nanoparticle," International Journal of Pharmacy \& Life Sciences, vol. 4, no. 7, 2013.

[54] M. Izadifar, M. E. Kelly, A. Haddadi, and X. Chen, "Optimization of nanoparticles for cardiovascular tissue engineering," Nanotechnology, vol. 26, no. 23, Article ID 235301, 2015.

[55] I. G. Zigoneanu, C. E. Astete, and C. M. Sabliov, "Nanoparticles with entrapped $\alpha$-tocopherol: synthesis, characterization, and controlled release," Nanotechnology, vol. 19, no. 10, Article ID 105606, 2008.

[56] H. Wang, Y. Zhao, Y. Wu et al., "Enhanced anti-tumor efficacy by co-delivery of doxorubicin and paclitaxel with amphiphilic methoxy PEG-PLGA copolymer nanoparticles," Biomaterials, vol. 32, no. 32, pp. 8281-8290, 2011.

[57] P. Le Corre, P. Le Guevello, V. Gajan, F. Chevanne, and R. Le Verge, "Preparation and characterization of bupivacaineloaded polylactide and polylactide-co-glycolide microspheres," International Journal of Pharmaceutics, vol. 107, no. 1, pp. 41-49, 1994. 

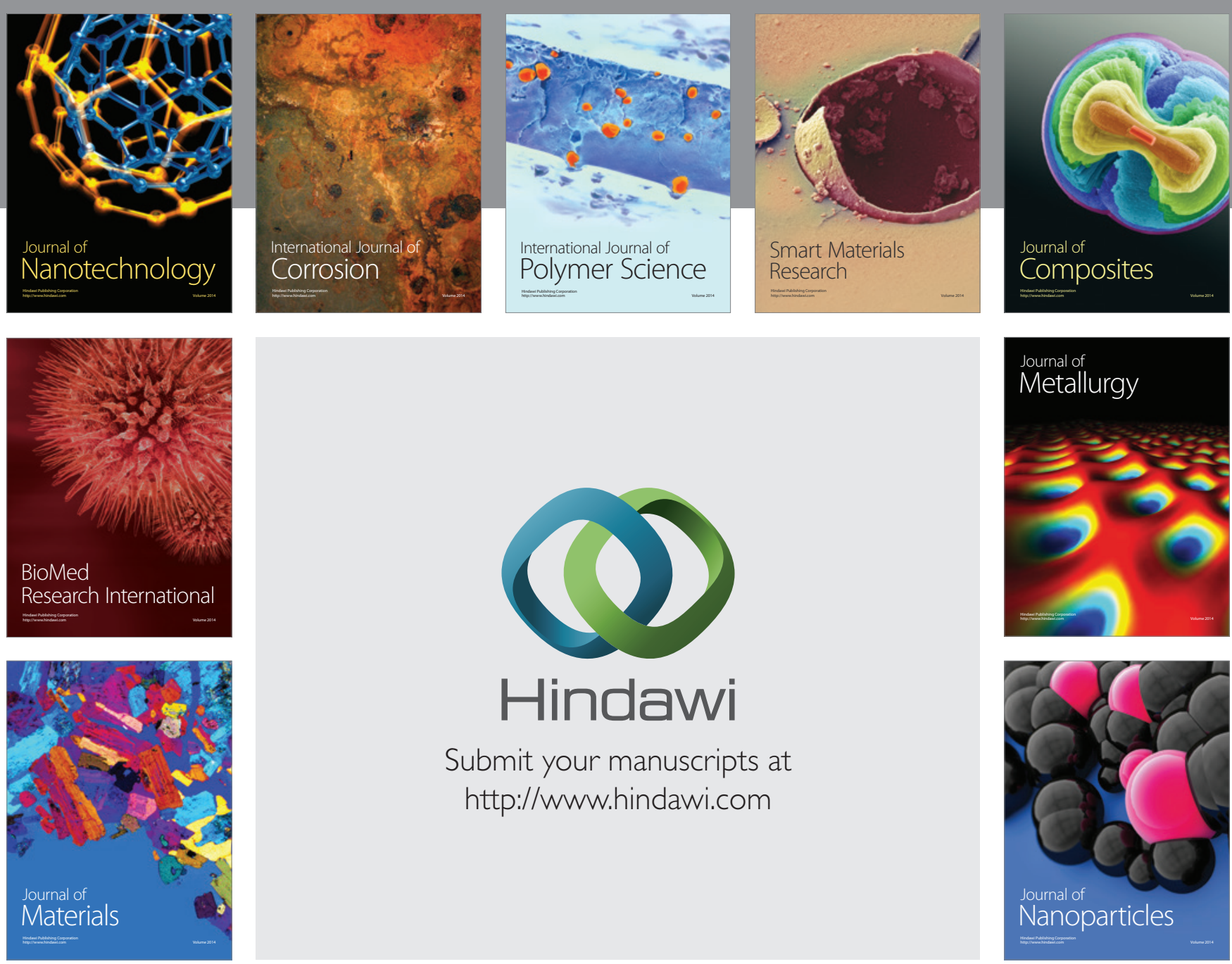

Submit your manuscripts at http://www.hindawi.com
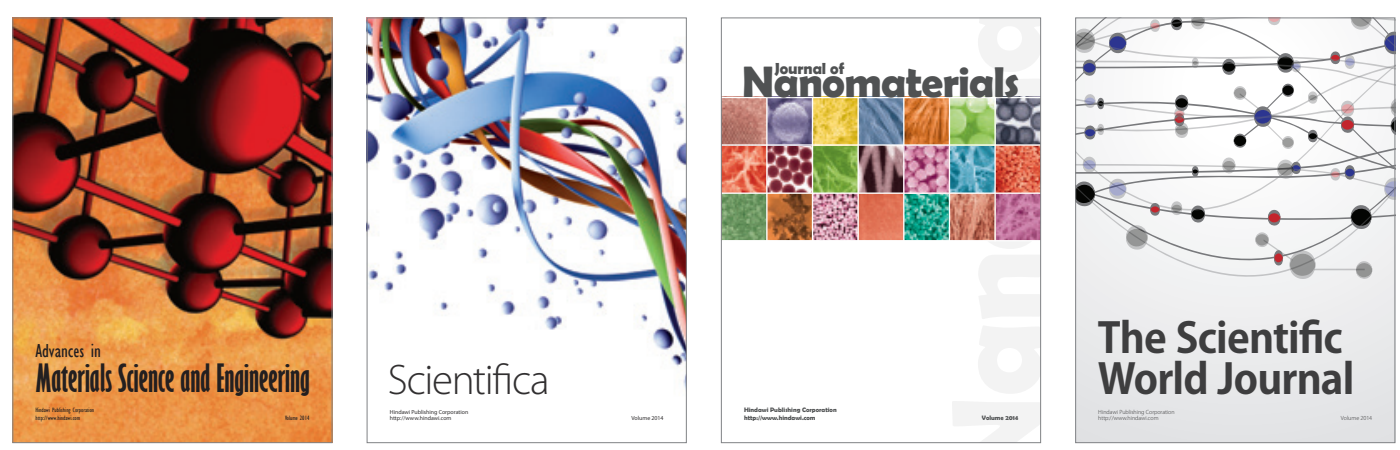

\section{The Scientific World Journal}
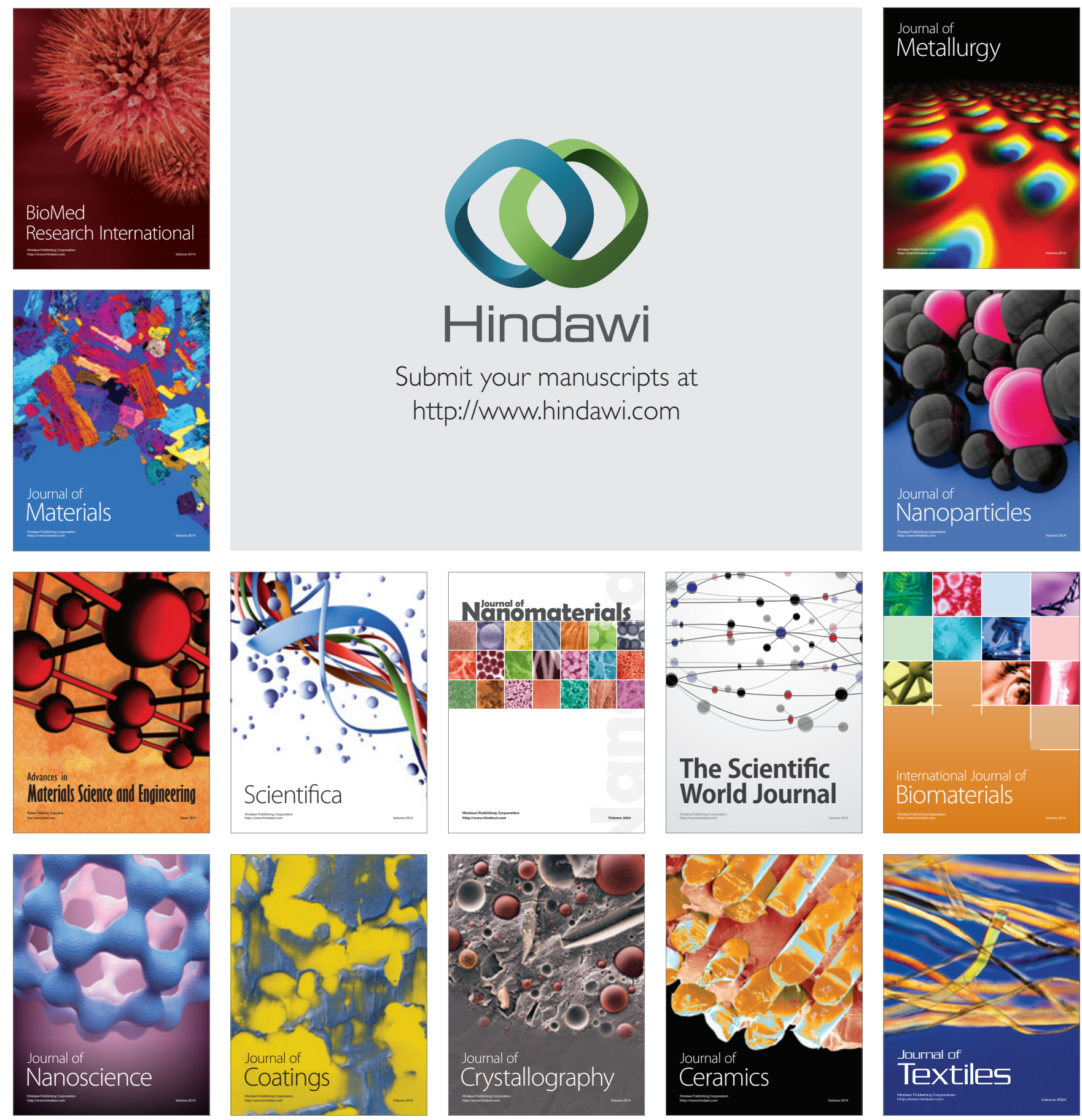